\title{
How acetaldehyde reacts with low molecular weight phenolics in white and red wines
}

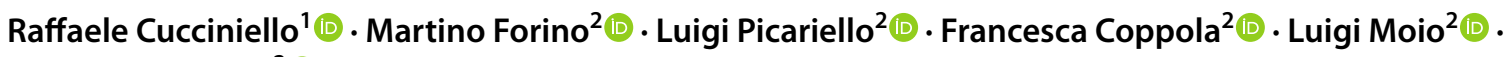 \\ Angelita Gambuti ${ }^{2}$ (I)
}

Received: 15 May 2021 / Revised: 26 July 2021 / Accepted: 31 July 2021 / Published online: 11 August 2021

(c) The Author(s) 2021

\begin{abstract}
Acetaldehyde is a key compound in determining wine color evolution and sensory properties. Major wine metabolites reactive to acetaldehyde are phenolic compounds, mainly flavan-3-ols and anthocyanins. Many studies have been conducted with the purpose of investigating acetaldehyde reactivity in model solutions, but very poor are the reports of its fate in real wines. By means of LC-HRESIMS and UV/Vis HPLC, red and white wines exposed to an excess of acetaldehyde were analyzed with a specific focus on low molecular weight phenolics. The chemical behavior of acetaldehyde turned out to be different in white and red wines. In white wines, it mainly mediated the formation of vinyl-flavan-3-ol derivatives, while in red wines it led to the formation of ethylidene-bridged red pigments. These latter positively enhanced the color properties of red wines. Conversely, in white wines, the formation of compounds, such as xanthylium ions, causing the undesired browning effects were not detected.
\end{abstract}

Keywords Acetaldehyde reactivity $\cdot$ Wine phenolics $\cdot$ Red wine $\cdot$ White wine $\cdot$ Wine color

\section{Introduction}

Acetaldehyde is the most abundant volatile aldehyde in wines. It can have a double origin either biological or chemical. During the early fermentation phase, acetaldehyde is formed by yeasts as a side product of the alcoholic fermentation, even if it is successively degraded by yeasts themselves or by malolactic bacteria. Chemically, acetaldehyde derives from the oxidation of ethanol occurring, after the fermentation, by the Fenton reaction [1]. It is a highly reactive aldehyde featuring both electrophilic and nucleophilic properties and, considering its high volatility, it is a flavor active molecule reminiscent of green grass, bruised apples and nuts with a sensory threshold of about $100 \mathrm{mg} / \mathrm{L}$. Indeed, acetaldehyde can react with other wine compounds including glycerol thus

Martino Forino

forino@unina.it

1 Department of Chemistry and Biology 'Adolfo Zambelli', University of Salerno, Via Giovanni Paolo II, 132, 84084 Fisciano, SA, Italy

2 Department of Agricultural Sciences, Section of Vine and Wine Sciences, University of Napoli 'Federico II', Viale Italia, 83100 Avellino, Italy leading to the formation of dioxolane and dioxane acetals responsible for wine oxidative spoilage [2].

At higher concentrations, it can be quite pungent with a negative impact on the wine quality. Still, in red, wines acetaldehyde can be even responsible for some beneficial reactions, involving mainly phenolics that could improve wine color stability and astringency over time $[3,4]$. Typical adducts formed by the reaction of acetaldehyde with phenolics consist of ethylidene-bridged compounds. Flavan3-ols, including catechin, epicatechin, proanthocyanidins, and anthocyanins are among the major wine polyphenols reactive to acetaldehyde. Considering that acetaldehyde protonation is the first step of the reaction, $\mathrm{pH}$ is a critical parameter to be taken into account. Low $\mathrm{pH}$ values around 2 determine high reaction rates. Consequently, wine $\mathrm{pH}$ usually stretching from 3 to 4 causes acetaldehyde to react with flavonoids more slowly. In this regard, several studies have been conducted in wine model solutions [5-8], while little is currently available in literature about the fate of acetaldehyde in real wines owing to the complexity of the product mixture deriving from the reaction with phenolics. It needs to be underlined that acetaldehyde rapidly reacts with bisulfites that are commonly added to wines as to prevent them from spoilage of either chemical or microbial origin. 
The product of such reaction is 1-hydroxyethanesulfonate [9]. In addition, when the concentration of sulfur dioxide is twice that of flavanols, reactions of acetaldehyde consuming catechin tend to cease [9]. This is the reason why in this study with the purpose of providing further insights into the acetaldehyde reactivity with phenolics in real wines, we decided to use wines with reduced amounts of bisulfites.

When anthocyanins and flavanols simultaneously occur, as in the case of red wines, the reaction of acetaldehyde with anthocyanins is slower than that with flavanols seemingly due to the better nucleophilicity of flavanol's position 8 and, to a lesser extent, of position 6 , too. Previous analyses have characterized a number of products of the reaction of acetaldehyde with catechin and malvidin-3-O-glucoside in wine model solution. Specifically, the most abundant product was identified as a catechin-ethyl-malvidin-3-Oglucoside in addition to other ethylidene-bridged oligomers constituted by up to four catechin units or by up to three catechin units containing also malvidin-3-O-glucoside in a terminal chain position [9]. Ethylidene-bridged oligomers containing flavanols and two anthocyanins terminal units have been detected as well [8]. Conversely, unlike flavanols, anthocyanins tend to form preferentially dimers $[10,11]$. To make the product mixture even more complex, vinyl flavan3-ols either as monomers or as subunits included in oligomers can also be formed [12].

Other anthocyanin-derived pigments detected in red wines are pyranoanthocyanins. They are formed by the reactions between anthocyanins and other wine metabolites, such as pyruvic acid and acetaldehyde, or with compounds extracted from grapes, such as cinnamic acids. When malvidin-3-O-glucoside reacts with pyruvic acid and acetaldehyde, vitisin A (1) [13] and vitisin B (2) [14] are, respectively, formed. Pyranoanthocyanins play an important role in wine ageing, as they are more stable in solution as opposed to flavanol-anthocyanin polymers that tend to precipitate over time [15]. Also, these pigments with their predominant red-orange hues differ from anthocyanins characterized by a red-purple color.

As stated above, in the face of several studies conducted on the reactivity of acetaldehyde with selected wine phenolics in model solutions, very few are the reports on such reactions in real wines. Although the role of anthocyanins has been extensively investigated, to the best of our knowledge, no studies focused on possible differences between the behavior of white and red wines when added with the same amount of acetaldehyde have been conducted yet. Hence, the present study was designed and carried out with the purpose of gaining preliminary information about the reactivity of acetaldehyde with low molecular weight phenolics in both white and red wines. Additionally, the outcome of such a study was expected to provide further insights into the color evolution of wines.

\section{Materials and methods}

\section{Chemicals and reagents}

Malvidin-3- $O$-glucoside chloride (>90\% HPLC) and (+)-catechin (99\% HPLC) standards, formic acid for LC-MS analysis and acetonitrile (hypergrade for LC-MS LiChrosolv ${ }^{\circledR}$ ) were purchased from Sigma-Aldrich (Milan, Italy). Aqueous solutions were prepared with Milli-Q water from Millipore (Bedford, MA, USA).

\section{Wine samples}

Aglianico red wine and Falanghina white wine, both produced in 2018 by the Taburno winery with a standard industry protocol, were added with $190 \mathrm{mg} / \mathrm{L}$ of pure acetaldehyde. Aglianico base parameters were: ethanol 13.37\%, pH 3.69, titratable acidity $5.4 \mathrm{~g} / 1$, volatile acidity $0.54 \mathrm{~g} / \mathrm{l}$. Falanghina base parameters were: ethanol $13.12 \%, \mathrm{pH} 3.42$, titratable acidity $6.6 \mathrm{~g} / \mathrm{l}$, volatile acidity $0.34 \mathrm{~g} / \mathrm{l}$. Free and total sulphur dioxide were below detection threshold [16]. The same Aglianico and Falanghina wines, without the addition of acetaldehyde, were used as control. Treated and untreated wines were stored in $5 \mathrm{~L}$ glass flasks hermetically closed in the dark at $20^{\circ} \mathrm{C}$ for one year.

\section{High-performance liquid chromatography analyses of anthocyanins in red wine samples}

The separation of the monomeric anthocyanins was performed according to the OIV method [17] of analysis using a HPLC Shimadzu LC10 ADVP apparatus (Shimadzu Italy, Milan), consisting of a SCL-10AVP system controller, two LC-10ADVP pumps, an SPD-M 10 AVP detector, and an injection system full Rheodyne model 7725 (Rheodyne, Cotati, CA). A column heating device set at $40{ }^{\circ} \mathrm{C}$ was used, with a $\mathrm{C} 18$ column, Waters Spherisorb column $(250 \times 4.6 \mathrm{~mm}, 4 \mu \mathrm{m}$ particles diameter $)$ with precolumn (Oiv.int). All the samples were filtered through $0.45 \mu \mathrm{m}$ filters (Durapore membrane filters, MilliporeIreland) into glass vials and immediately injected into the HPLC system. A $50 \mu \mathrm{L}$ loop was used. Elution was carried out using a flow rate of $0.80 \mathrm{~mL} / \mathrm{min}$. Eluents were: solvent A consisting of water milli-Q (Sigma-Aldrich)/ formic acid (Sigma-Aldrich $\geq 95 \%$ )/acetonitrile (SigmaAldrich $\geq 99.9 \%)(87: 10: 3 \mathrm{v} / \mathrm{v} / \mathrm{v})$ and, solvent B consisting of water/formic acid/acetonitrile $(40: 10: 50 \mathrm{v} / \mathrm{v} / \mathrm{v})$. The following gradient was used: zero-time conditions $94 \% \mathrm{~A}$ and $6 \% \mathrm{~B}$; after 15 min the pumps were adjusted to $70 \% \mathrm{~A}$ and $30 \% \mathrm{~B}$, at $30 \mathrm{~min}$ to $50 \% \mathrm{~A}$ and $50 \% \mathrm{~B}$, at $35 \mathrm{~min}$ to $40 \% \mathrm{~A}$ and $60 \% \mathrm{~B}$; at $42 \mathrm{~min}$ through the end of analysis, 
to $94 \% \mathrm{~A}$ and $6 \% \mathrm{~B}$. For calibration the external standard method was used: the calibration curve was plotted for the malvidin-3-O-glucoside on the basis of peak area. The concentration of the following monomeric anthocyanins was determined in red wine used as control, after 1 year of incubation, and expressed as $\mathrm{mg} / \mathrm{L}$ : delphinidin 3-O-glucoside (39.05 \pm 1.46$)$; cyanidin $3-O$-glucoside $(2.82 \pm 0.13)$; petunidin-3-O-glucoside $(49.68 \pm 1.07)$; peonidin $3-O$-glucoside $(22.59 \pm 1.01)$; malvidin-3-O-glucoside $(266.77 \pm 14.02)$; peonidin-3-(6 ${ }^{\mathrm{II}}$-acetyl)- $O$-glucoside $(8.60 \pm 0.40)$; malvidin 3 -(6 $6^{\mathrm{II}}$-acetyl)- $O$-glucoside $(5.84 \pm 0.20)$; malvidin $3-\left(6^{\mathrm{II}}\right.$ coumaroyl)- $O$-glucoside $(34.54 \pm 0.43)$. In red wine added with acetaldehyde, after 1 year of incubation, only malvidin-3-O-glucoside $(16.55 \pm 0.25 \mathrm{mg} / \mathrm{L})$ was quantified. The remaining anthocyanins were detected only in traces. The concentration was expressed as $\mathrm{mg} / \mathrm{L}$ of malvidin 3-O-glucoside. All experiments were carried out in duplicate and two analytical replicas were performed.

\section{$\mathrm{HPLC/high-resolution} \mathrm{electrospray} \mathrm{ionization} \mathrm{mass}$ spectrometry (LC-HR ESIMS) of white wines}

Chromatographic separation of white wines as such was carried out on a C18 Kinetex column $(2.6 \mu \mathrm{m}, 2.10 \times 100 \mathrm{~mm}$, Phenomenex USA). Eluents were: solvent A consisting of water milli-Q (Sigma-Aldrich)/formic acid (SigmaAldrich $\geq 95 \%$ )/acetonitrile (Sigma-Aldrich $\geq 99.9 \%)(96: 1: 3$ $\mathrm{v} / \mathrm{v} / \mathrm{v}$ ) and, solvent B consisting of water/formic acid/acetonitrile $(49: 1: 50 \mathrm{v} / \mathrm{v} / \mathrm{v})$. The following gradient was used: zero-time conditions $94 \% \mathrm{~A}$ and $6 \% \mathrm{~B}$; after $15 \mathrm{~min}$ the pumps were adjusted to $70 \% \mathrm{~A}$ and $30 \% \mathrm{~B}$; at $30 \mathrm{~min}$ to $50 \%$ $\mathrm{A}$ and $50 \% \mathrm{~B}$; at $35 \mathrm{~min}$ to $40 \% \mathrm{~A}$ and $60 \% \mathrm{~B}$; at $41 \mathrm{~min}$ through the end of analysis, to $94 \% \mathrm{~A}$ and $6 \% \mathrm{~B}$. Flow rate was $0.3 \mathrm{~mL} / \mathrm{min}$ and injection volume $0.5 \mu \mathrm{L}$. LC-HR ESIMS experiments in the positive and negative ion mode were performed on an Agilent 1260 Infinity II HPLC quaternary system coupled to a linear ion trap LTQ Orbitrap XL hybrid Fourier transform MS (FTMS) instrument equipped with an ESI ION MAX source (Thermo-Fisher). The following source settings were used for HR-MS (mass range $\mathrm{m} / \mathrm{z}, 100-2000$ ): spray voltage $4,5 \mathrm{kV}$, capillary temperature $300{ }^{\circ} \mathrm{C}$, capillary voltage $15 \mathrm{~V}$, sheath gas 20 and auxiliary gas 21 (arbitrary units), and tube lens voltage $140 \mathrm{~V}$, and $25 \%$ collision energy. Calculation of elemental formulae was conducted using Xcalibur software $\mathrm{v}$ 2.0.7. with a mass tolerance constrain of $5 \mathrm{ppm}$. For quantitative purposes, extracted ion chromatograms (XICs) of all the selected analytes were obtained with a mass tolerance of $5 \mathrm{ppm}$.

$(+)$-catechin and (-)-epicatechin standards were used for quantitative determinations by plotting a calibration curve on the basis of peak areas (triplicate injections) at six levels of concentrations $(0.1,0.5,1.0,5.0,10.0$, and $20.0 \mathrm{mg} / \mathrm{L})$. Epicatechin, Catechin- and epicatechin-derivatives detected in white wines were quantitatively determined by assuming that their molar responses were similar to that of $(+)$-catechin. The calibration curve equation for $(+)$-catechin standard was $\mathrm{y}=0.19232 \mathrm{x}-0.0023$ and its linearity was $\mathrm{R}^{2}=0,9953$. LOD and LOQ were 0.36 and $1.09 \mathrm{mg} / \mathrm{L}$, respectively. The calibration curve for (-)-epicatechin standard was $\mathrm{y}=0.1476 \mathrm{x}+0.0115$ and its linearity was $\mathrm{R}^{2}=0.9959$. LOD and LOQ were 0.34 and $1.02 \mathrm{mg} / \mathrm{L}$.

\section{HPLC and LC-HR ESIMS analyses of red wines}

Before LC-HR ESIMS analyses, red wines were preliminary subjected to HPLC separation at a semi-preparative scale using the same elution conditions described above for the identification of anthocyanins, except the employed column that was a Luna $10 \mathrm{u}$ Column and the flow rate that was set at $1.8 \mathrm{~mL} / \mathrm{min}$. From this chromatographic separation, five fractions were collected: A (from 7 to $24 \mathrm{~min}$ ), B (from 24 to $25 \mathrm{~min}$ ), C (from 25 to $26 \mathrm{~min}$ ), $\mathrm{D}$ (from 26 to $28 \mathrm{~min}$ ), and $\mathrm{E}$ (from $28 \mathrm{~min}$ to end of the run at $42 \mathrm{~min}$ ). Each collected fraction was successively concentrated to $1 \mathrm{ml}$ final volume under nitrogen, and finally analyzed by LC-HR ESIMS in the positive and negative ion mode using the same parameters reported above for the analyses of white wines except the elution gradient. Eluents were: solvent A consisting of water milli-Q (Sigma-Aldrich)/ formic acid (Sigma-Aldrich $\geq 95 \%$ )/acetonitrile (SigmaAldrich $\geq 99.9 \%)(87: 10: 3) \mathrm{v} / \mathrm{v} / \mathrm{v}$ and, solvent B consisting of water/formic acid/acetonitrile (40:10:50) $\mathrm{v} / \mathrm{v} / \mathrm{v}$. The following gradient was used: zero-time conditions $94 \% \mathrm{~A}$ and $6 \% \mathrm{~B}$; after 20 min the pumps were adjusted to $70 \% \mathrm{~A}$ and $30 \% \mathrm{~B}$; at $40 \mathrm{~min}$ to $50 \% \mathrm{~A}$ and $50 \% \mathrm{~B}$; at $50 \mathrm{~min}$ to $40 \% \mathrm{~A}$ and $60 \%$ $\mathrm{B}$; at $51 \mathrm{~min}$ through the end of the analysis (65 $\mathrm{min}$ ) to $94 \%$ $\mathrm{A}$ and $6 \% \mathrm{~B}$.

\section{CIELAB coordinates and color intensity and hue.}

The CIELAB parameters $\left(L^{*} . a^{*} . b^{*}\right)$ were determined using the software Panorama (PANORAMA SOFTWARE UPGRADE PATH), following the recommendations of the Commission Internationale de L'Eclariage (CIE). Color differences $(\Delta \mathrm{E} / \mathrm{ab})$ were calculated as the Euclidean distance between two points in the 3D space defined by $\mathrm{L}^{*}, \mathrm{a}^{*}$, and $\mathrm{b}^{*}$. Color intensity (Abs $420 \mathrm{~nm}+\mathrm{Abs} 520 \mathrm{~nm}+\mathrm{Abs}$ $620 \mathrm{~nm}$ ) and hue (Abs $420 \mathrm{~nm} / \mathrm{Abs} 520 \mathrm{~nm}$ ) were determined spectrophotometrically using a UV spectrophotometer. All analyses were carried out in duplicate.

\section{Results and discussion}

Each finished red and white wine was subdivided into two aliquots, respectively: one was used as control and the other was treated with an excess of acetaldehyde. Treated wines 
and control wines were stored under the same conditions for 1 year. This was a reasonable stretch of time usually elapsing for a typical wine from bottling to consumption. The purpose of the addition of an excess of acetaldehyde was to favor the formation of products deriving from the reaction between the aldehyde and wine phenolics at a concentration high enough to be detected and analyzed. These reactions, though only a part of those taking place during wine fermentation and oxidation, are crucial for the stability of the wine color especially during the first years of aging.

As for white wines, information on the effect of acetaldehyde on phenolics is still quite scarce. In our study, the comparison between the treated and untreated white wines allowed to shed some light on the evolution of phenolics following the addition of acetaldehyde. This was achieved by means of LC-HR ESIMS.

In red wines, the addition of acetaldehyde induced a massive formation of polymeric compounds. This was ascertained when a preliminary HPLC-based analysis was conducted. Unlike the control wine, the occurrence of polymeric pigments in the fortified wine was reflected by a significant drift of the UV/vis chromatogram baseline recorded at $518 \mathrm{~nm}$. Hence, to get qualitative insights into the complex polymeric fraction of the treated wines, a HPLC-based chromatographic separation at a semi-preparative scale was initially performed, as reported in Experimental. Five fractions were collected (A-E) and each of them was subjected to LC-HR ESIMS.

\section{Analysis of white wines}

Samples of treated and untreated white wines were analyzed by LC-HR ESIMS after one year of incubation. The comparison of the total ion chromatograms (TIC) of the white wine used as control (sample \#1) and the white wine (sample \#2) added with the aldehyde brought to light a significant decrease of the relative abundance of some chromatographic peaks along with a concomitant increase of others (Fig. 1). Discrepancies in terms of peak intensities were ascertained by measuring the areas of the ion peaks evidently differing in the chromatograms of sample \#1 and \#2. In more detail, in the TIC of sample \#2 we observed the increment of peaks eluting at $12.40,13.32,14.70,14.90$, and $16.37 \mathrm{~min}$, respectively. Peaks eluting at $12.85,15.12,16.20$, and 20.00 remained approximately unaffected; whilst, peaks at 9.65, $11.15,13.51$ and $14.50 \mathrm{~min}$, respectively, appeared to be less intense when compared to the TIC of sample \#1 (Table 1). By comparison with pure standards, the two peaks eluting at 11.15 and $13.51 \mathrm{~min}(\mathrm{~m} / \mathrm{z} 289)$ were attributed to catechin and epicatechin (Fig. 2), respectively. On account of previous reports, the peaks eluting at $9.65 \mathrm{~min}(\mathrm{~m} / \mathrm{z}, 577)$ and $14.50(\mathrm{~m} / \mathrm{z} 865)$ were assigned to a procyanidin dimer and trimer, respectively (Fig. 2) [18, 19]. The remaining peaks were reasonably interpreted as the result of the reaction between wine flavanols and acetaldehyde. More specifically, on the basis of their retention times and $\mathrm{m} / \mathrm{z}$ values (315), the two ion peaks at 14.90 and 16.37 min were assigned to a vinyl-catechin and vinyl-epicatechin derivative (Fig. 2), respectively. The two ion peaks differed indeed from either catechin or epicatechin by 26 u.m.a., corresponding to a $\mathrm{C}_{2} \mathrm{H}_{2}$-moiety [4]. Such vinyl derivatives are likely the result of the dehydration of ethyl alcohol-flavan-3-ol adducts deriving from the nucleophilic attack of the flavan-3-ol $\mathrm{C} 8$ or, to a lesser extent, C6 to protonated acetaldehyde. In the treated wines, we did not detect ethyl alcohol-flavan3-ol adducts, but peaks at 12.85 and $15.12 \mathrm{~min}(\mathrm{~m} / \mathrm{z} 359)$ were likely the result of these latter derivatives with an extra vinyl moiety (Fig. 2) linked to either 6 or 8 position. Hence, these molecules were identified as ethyl alcoholvinyl-catechin adducts (12.85 $\mathrm{min})$ and ethyl alcohol-vinylepicatechin adducts (15.12 min). It is reasonable to infer that such derivatives underwent dehydration thus turning into

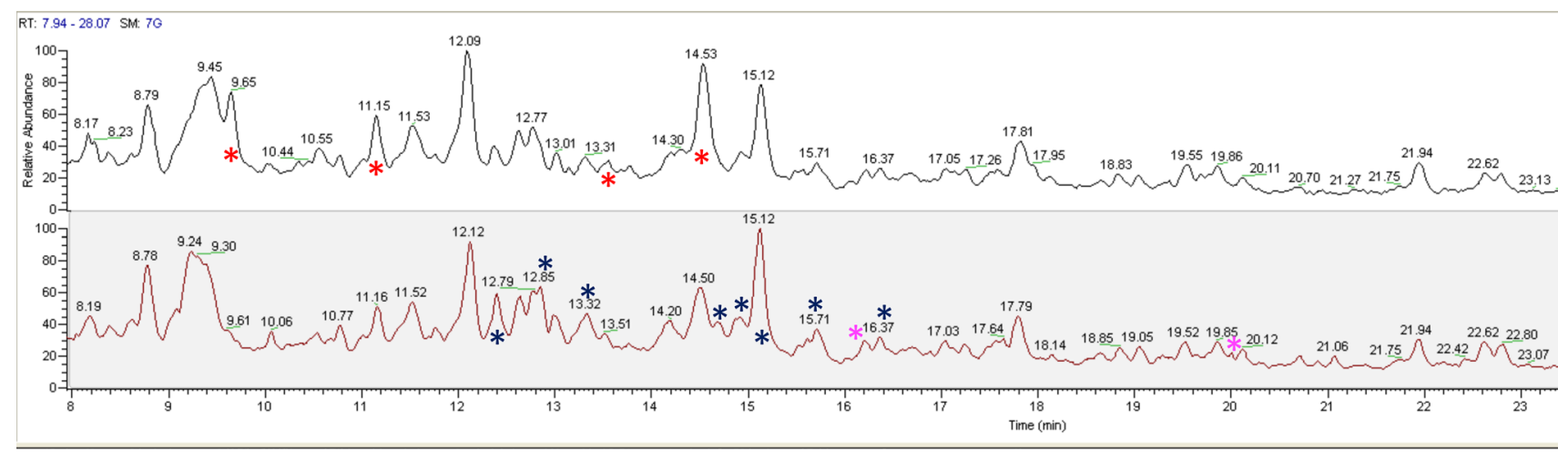

Fig. 1 Total ion current chromatograms of a sample of control white wine (above) and of a sample of white wine treated with acetaldehyde. Red and blue asterisks indicate ion peaks that decreased or increased, respectively, after the addition of acetaldehyde (Table 1); magenta asterisks indicate peaks that seem to have been not affected by the addition of acetaldehyde 
Table 1 Compounds whose concentrations was modified by the addition of acetaldehyde to white wines. Untreated wines (sample \#1); treated wines (sample \#2)

\begin{tabular}{|c|c|c|c|c|c|c|}
\hline Retention time & {$[\mathrm{M}-\mathrm{H}]^{-} m / z ; \Delta(\mathrm{ppm})$} & Formula & Compound & $\mathrm{mg} / \mathrm{L}$ in sample \#1 & $\mathrm{mg} / \mathrm{L}$ in sample \#2 & $\begin{array}{l}\text { Fragment ions } \\
{[\mathrm{MS} / \mathrm{MS}](\mathrm{m} / \mathrm{z})}\end{array}$ \\
\hline $9.65 \mathrm{~min}$ & $577.1345 ; \Delta=0.741$ & $\mathrm{C}_{30} \mathrm{H}_{25} \mathrm{O}_{12}$ & Procyanidin dimer & $7.10 \pm 0.04$ & $1.05 \pm 0.02$ & $407,289,245$ \\
\hline $11.15 \mathrm{~min}$ & $289.0711 ; \Delta=1.610$ & $\mathrm{C}_{15} \mathrm{H}_{13} \mathrm{O}_{6}$ & catechin & $2.02 \pm 0.05$ & $0.92 \pm 0.03$ & $\begin{array}{l}245,203,159,151,137 \\
125,109\end{array}$ \\
\hline $12.40 \mathrm{~min}$ & 429.2121 & n.a & Undetermined & - & - & - \\
\hline $12.85 \mathrm{~min}$ & $359.1143 ; \Delta=1.876$ & $\mathrm{C}_{19} \mathrm{H}_{19} \mathrm{O}_{7}$ & $\begin{array}{l}\text { ethyl alcohol-vinyl- } \\
\text { catechin }\end{array}$ & $2.84 \pm 0.03$ & $2.93 \pm 0.04$ & 341,313 \\
\hline $13.32 \mathrm{~min}$ & $603.1496 ; \Delta=-0.137$ & $\mathrm{C}_{32} \mathrm{H}_{27} \mathrm{O}_{12}$ & $\begin{array}{l}\text { vinyl-Procyanidin } \\
\text { dimer }\end{array}$ & $0.12 \pm 0.08$ & $1.95 \pm 0.1$ & 467,289 \\
\hline $13.51 \mathrm{~min}$ & $289.0709 ; \Delta=0.987$ & $\mathrm{C}_{15} \mathrm{H}_{13} \mathrm{O}_{6}$ & epicatechin & $0.73 \pm 0.02$ & $0.36 \pm 0.05$ & $\begin{array}{l}245,203,187,151,137 \\
125,121,109\end{array}$ \\
\hline $14.50 \mathrm{~min}$ & $865.1973 ; \Delta=-1.395$ & $\mathrm{C}_{45} \mathrm{H}_{37} \mathrm{O}_{18}$ & Procyanidin trimer & $0.25 \pm 0.03$ & $0.04 \pm 0.02$ & 577,289 \\
\hline $14.70 \mathrm{~min}$ & $\begin{array}{l}\text { 603. 1496; } \\
\quad \Delta=-0.137\end{array}$ & $\mathrm{C}_{32} \mathrm{H}_{27} \mathrm{O}_{12}$ & $\begin{array}{l}\text { vinyl-Procyanidin } \\
\text { dimer }\end{array}$ & $0.08 \pm 0.04$ & $2.03 \pm 0.1$ & 467,289 \\
\hline $14.90 \mathrm{~min}$ & $315.0866 ; \Delta=0.874$ & $\mathrm{C}_{17} \mathrm{H}_{15} \mathrm{O}_{6}$ & vinyl-catechin & $0.05 \pm 0.01$ & $0.57 \pm 0.03$ & 287 \\
\hline $15.12 \mathrm{~min}$ & $359.1143 ; \Delta=1.876$ & $\mathrm{C}_{19} \mathrm{H}_{19} \mathrm{O}_{7}$ & $\begin{array}{l}\text { ethyl alcohol-vinyl- } \\
\text { epicatechin }\end{array}$ & $1.48 \pm 0.1$ & $1.69 \pm 0.05$ & 341 \\
\hline $16.20 \mathrm{~min}$ & $341.1036 ; \Delta=1.579$ & $\mathrm{C}_{19} \mathrm{H}_{17} \mathrm{O}_{6}$ & 6,8-divinyl-catechin & $1.11 \pm 0.08$ & $1.08 \pm 0.05$ & 323,295 \\
\hline $16.37 \mathrm{~min}$ & $315.0866 ; \Delta=0.874$ & $\mathrm{C}_{17} \mathrm{H}_{15} \mathrm{O}_{6}$ & vinyl-epicatechin & $0.04 \pm 0.02$ & $0.59 \pm 0.09$ & 287 \\
\hline $20.00 \mathrm{~min}$ & $341.1036 ; \Delta=1.579$ & $\mathrm{C}_{19} \mathrm{H}_{17} \mathrm{O}_{6}$ & 6,8-divinyl-epicatechin & $2.32 \pm 0.01$ & $2.12 \pm 0.02$ & 295 \\
\hline
\end{tabular}<smiles>Oc1cc(O)c2c(c1)O[C@H](c1ccc(O)c(O)c1)[C@H](O)C2</smiles>

catechin<smiles>C=Cc1c(O)cc(O)c2c1O[C@H](c1ccc(O)c(O)c1)[C@H](O)C2</smiles><smiles>Oc1cc(O)c2c(c1)O[C@H](c1ccc(O)c(O)c1)[C@H](O)C2</smiles>

epicatechin<smiles>C[C@@H](O)c1c(O)cc(O)c2c1O[C@H](c1ccc(O)c(O)c1)[C@H](O)C2</smiles>

$\mathrm{OH}$<smiles>Oc1cc(O)c2c(c1)O[C@H](c1ccc(O)c(O)c1)[C@H](O)C2c1c(O)cc(O)c2c1O[C@H](c1ccc(O)c(O)c1)[C@H](O)C2</smiles>

procyanidin dimer<smiles>Cc1cc([C@H]2Oc3cc(O)cc(O)c3C(c3c(O)cc(O)c4c3O[C@H](c3ccc(O)c(O)c3)[C@H](O)C4c3c(O)cc(O)c4c3O[C@H](c3ccc(O)c(O)c3)[C@H](O)C4)[C@H]2O)ccc1O</smiles>

procyanidin trimer

Fig. 2 Stereostructures of compounds detected by LC-HR ESIMS in white wines 
6,8-divinyl-catechin (16.20 $\mathrm{min} ; \mathrm{m} / \mathrm{z} 341)$ and 6,8-divinylepicatechin (20.00 $\mathrm{min} ; \mathrm{m} / \mathrm{z} 341)$, respectively. Finally, the two ion peaks eluting at 13.32 and 14.70 min both sharing the same $\mathrm{m} / \mathrm{z}$ value of 603 were assigned to dimeric vinyl-procyanidins. Only the peak at 12.40 remained undetermined. Interestingly, no ion peaks corresponding to possible ethylidene linked flavan-3-ols oligomers were detected.

Additionally, quantitative analyses were conducted by LC-HR ESIMS as reported in Experimental. Quantitation of catechin and epicatechin was carried out by comparison with pure standards. All of the other identified flavanol derivatives were quantified by assuming they had the same molar response as catechin. On the basis of our results, a significant decrease of over $80 \%$ was observed for procyanindin dimers and trimers, confirming previous reports according to which procyanidin $\mathrm{B}_{2}$ in model solution added with acetaldehyde was no longer detectable after 15 days [5]. Catechin and epicatechin appeared to be reduced by approximately $50 \%$ in the treated wines (sample \#2) in comparison to untreated ones (sample \#1). At the same time, though, the relative abundances of ethyl alcohol-vinyl-flavan-3-ols and of divinyl-flavan-3-ols were comparable in the treated and untreated wines, as opposed to vinyl-flavan-3-ols that remarkably increased in sample \#2. This datum likely reflects the complex evolution of flavanols mixtures characterized by $\mathrm{C}-\mathrm{C}$ bond breaking and forming that take place even under the wine mild conditions $[5,7,8]$.

It needs to be underlined that several studies have been conducted on the reactivity of flavan-3-ols towards acetaldehyde [9], in which the major observed products were oligomers, mainly dimers, consisting of catechin and epicatechin linked by an ethylidene bridge. However, to the best of our knowledge, experimental studies on the reactivity of flavan-3-ols with acetaldehyde have been conducted mainly in wine like solutions, while quite limited are the reports on real wines. In our studies, in white wines treated with an excess of acetaldehyde, we detected the occurrence of a number of newly formed products as reported in Table 1, but no ethylidene linked oligomers of flavanols. On the basis of our MS-based analyses, the detected compounds turned out to be all vinyl adducts of flavan-3-ols. According to the proposed mechanism of reaction ${ }^{6}$, such vinyl adducts can have a double origin. As already discussed above, they can derive from the dehydration of ethyl alcohol-flavan-3-ol adducts initially obtained from the reaction between acetaldehyde and flavan-3-ol at either position 8 or 6 , even if this latter position appears to be less reactive due to steric hindrance. Alternatively, the formation of vinyl derivatives can be the result of the depolymerization of ethylidene linked flavan3-ol oligomers. In this case, we can hypothesize that if such oligomers had been likely formed in the first place, their breakdown occurred as well. It has been in fact proposed that the rate of ethylidene-bridged flavan-3-ol formation is comparable to its rate of breakdown [4]. Hence, the depolymerized products could rearrange to form vinyl flavan3 -ols. Interestingly, a comparison of the relative abundance of flavanol derivatives between the treated and untreated wines brought to light substantial equal levels of those adducts featuring a double substitution at position 6 and 8 (ethyl alcohol-vinyl-(epi)catechin and divinyl-(epi)-catechin). This would be of some interest and worth to be fully investigated in model solutions to clarify their origin and rate of formation. It should also be taken into account that a possible source of such compounds may be due to yeasts metabolisms and this would pave the way towards microbiological studies, too. Finally, our results under the enological point of view suggest that in white wines flavanols tend to react with acetaldehyde, which in turn will be not initially involved in other reactions leading to the formation of offflavors including cyclic acetals and sotolon [20].

Our results highlight that, regardless of the origin of acetaldehyde in wine, flavanols in white wine are helpful to quench this highly reactive carbonyl by affording more stable compounds. Therefore, on one hand the "catechol" B-ring of flavonoids can be oxidized by quinones through electron transfer reactions leading to flavanoid quinones, precursors of browning products, but on the other they can prevent the negative off-flavors deriving from acetaldehyde produced during oxidation [21].

\section{Analysis of red wines}

After 1 year of incubation, the different evolutions of the wine used as control and the one added with acetaldehyde were suggested by their relative HPLC chromatograms registered at $1=518 \mathrm{~nm}$. As mentioned above, unlike the control, the chromatogram of the treated wine was characterized by a significant drift of the baseline likely due to a massive formation of polymeric pigments. Initially, the official OIV method [17] was applied to analyzed the two wines. In the untreated wine, a number of anthocyanins was identified and quantified, as reported in Experimental. Conversely, in the wine fortified with acetaldehyde only malvidin-3-O-glucoside could be detected. This datum confirmed that acetaldehyde had caused a dramatic loss of monomeric anthocyanins. In this regard, Dallas and colleagues [5] suggested that, in addition to malvidin-3-Oglucoside, two new colored compounds, obtained from the reaction of peonidin-3-O-glucoside and the procyanidin-acetaldehyde adduct, were simultaneously observed after $1 \mathrm{~h}$, they increased in 2 days and then began to decrease and were no longer detectable after 10 days. It is possible that the same phenomenon occurred in the wines used in our study. To better investigate the wine added with acetaldehyde, a preliminary semi-preparative HPLC separation of this wine was carried out and five 
fractions (A-E) collected. Each of these fractions was then analyzed by LC-HR ESIMS. By means of LC-MS, in fraction A, we detected an ion peak at $\mathrm{m} / \mathrm{z} 493$ attributable to malvidin-3-O-glucoside (Fig. 3, Table 2, SI 1a), while peonidin-3-O-glucoside $(\mathrm{m} / \mathrm{z} 463)$ was present just in traces (SI 1b). This was consistent with what initially observed by the HPLC-based analysis performed according to the OIV method.

The LC-MS spectrum of fraction B contained two ion peaks at $\mathrm{m} / \mathrm{z} 561$ and 517 attributed to two pyranomalvidin-3-O-glucosides, namely vitisin $\mathrm{A}$ and $\mathrm{B}$, respectively (Fig. 3; SI 2) [22, 23]. Two ion peaks centered at $m / z, 809$ in fraction $\mathrm{C}$ led us to infer the presence of dimers constituted by a malvidin-3-O-glucoside unit linked to a catechin unit $(\mathrm{Rt}=18.17 \mathrm{~min})$ and an epicatechin unit $(\mathrm{Rt}=18.97 \mathrm{~min})$, by an ethylidene bridge (SI 3). A mixture of diastereoisomers constituted by ethylidene dimers involving an anthocyanin unit and either catechin or epicatechin is not to be ruled out, considering that the ethylidene subunit deriving from acetaldehyde can connect the 8 position of the anthocyanin with either the 8 or 6 position of either flavan-3-ol (Fig. 3) [11].
In the LC-MS spectrum of fraction D, there was an ion peak that was attributed to a dimer constituted by one malvidin -3-O-glucoside in its flavylium ion form and one malvidin -3- $O$-glucoside unit in its pseudobase form linked by an ethylidene bridge ( $\mathrm{m} / \mathrm{z}$ 1029) (Fig. 3) [24].

Eventually, in E, some ion peaks turned out to be associated to a polymeric fraction eluting from 59 to $63 \mathrm{~min}$ (SI 4). These peaks were centered at $m / z 1029$ (the same as that contained in fraction D), 1097, and 1125, respectively. In Table 2, the above ion peaks are listed along with their molecular formulas and tentative chemical identification based on data available in literature [7-9] (Table 3).

Finally, vinyl-(epi)catechin and (epi)catechin-ethyl(epi)catechin derivatives were identified in the LC-HR ESIMS spectra of fraction A of the treated wines acquired in the negative ion mode. As discussed for white wines, the ion peaks at $m / z, 315$ were attributed to vinyl-catechin $(\mathrm{Rt}=14.82 \mathrm{~min})$, and to vinyl-epicatechin $(\mathrm{Rt}=16.61 \mathrm{~min})$. Additionally, an ion peak at $m / z 605.1651\left(\mathrm{C}_{32} \mathrm{H}_{29} \mathrm{O}_{12}{ }^{-}\right.$; $\Delta=-4.313 ; \mathrm{Rt}=13.95 \mathrm{~min}$ ) was indicative of the formation of (epi)catechin-ethyl-(epi)catechin adducts [6].<smiles></smiles>

malvidin-3-O-glu<smiles></smiles>

malvidin-3-O-glu-ethyl-(epi)catechin<smiles></smiles>

peonidin-3-O-glu

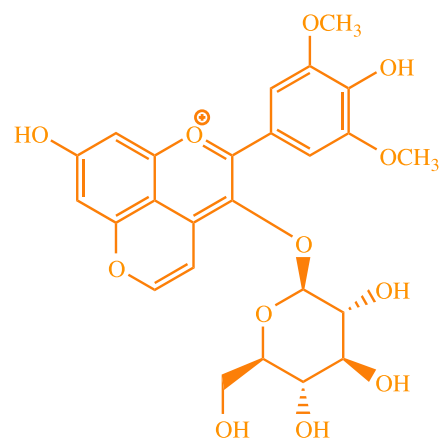

vitisin $\mathrm{B}$

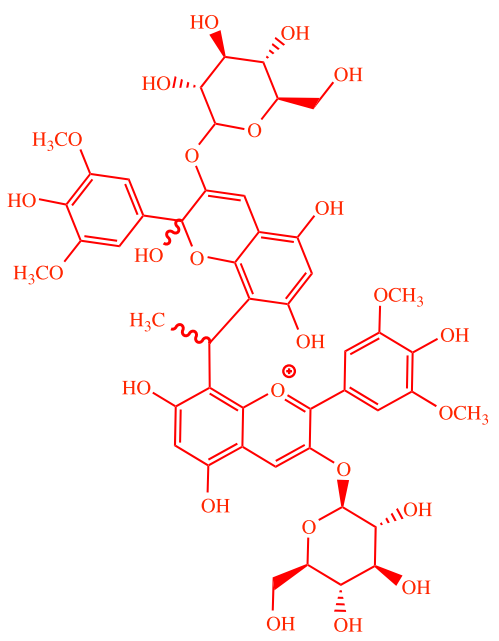

malvidin-3-O-glu-ethyl-malvidin-3-O-glu

Fig. 3 Stereostructures of pigments detected by LC-HR ESIMS in red wines 
Table 2 Compounds detected by LC-HR ESIMS (positive ion mode) in fractions A-E obtained from a red wine sample added with acetaldehyde

\begin{tabular}{|c|c|c|c|c|}
\hline $\begin{array}{l}\text { Fraction } \\
\text { Retention time }\end{array}$ & {$[\mathrm{M}]^{+} m / z ; \Delta(\mathrm{ppm})$} & Formula & Compound & $\begin{array}{l}\text { Fragment ions } \\
{[\mathrm{MS} / \mathrm{MS}](\mathrm{m} / \mathrm{z})}\end{array}$ \\
\hline \multicolumn{5}{|l|}{ A } \\
\hline $12.47 \mathrm{~min}$ & $463.1259 ; \Delta=5.208$ & \multirow[t]{2}{*}{$\mathrm{C}_{22} \mathrm{H}_{23} \mathrm{O}_{11}{ }^{+} \mathrm{C}_{23} \mathrm{H}_{25} \mathrm{O}_{12}{ }^{+}$} & Peonidin-3-O-glucoside & 301 \\
\hline $13.39 \mathrm{~min}$ & $493.1326 ; \Delta=-3.818$ & & Malvidin-3-O-glucoside & 331 \\
\hline \multicolumn{5}{|l|}{ B } \\
\hline $15.64 \mathrm{~min}$ & $561.1227 ; \Delta=-2.106$ & $\mathrm{C}_{26} \mathrm{H}_{25} \mathrm{O}_{14}^{+}$ & Vitisin A & 399 \\
\hline $17.15 \mathrm{~min}$ & $517.1317 ; \Delta=-4.491$ & $\mathrm{C}_{25} \mathrm{H}_{25} \mathrm{O}_{12}^{+}$ & Vitisin B & 355 \\
\hline \multicolumn{5}{|l|}{$\mathrm{C}$} \\
\hline $18.17 \mathrm{~min}$ & $809.2274 ; \Delta=-1.644$ & $\mathrm{C}_{40} \mathrm{H}_{41} \mathrm{O}_{18}^{+}$ & Malvidin-3-O-glucoside-ethyl- catechin & $647,519,357$ \\
\hline $18.97 \mathrm{~min}$ & & & Malvidin-3-O-glucoside-ethyl-epicatechin & $647,519,357$ \\
\hline \multicolumn{5}{|l|}{$\mathrm{D}$} \\
\hline $41.53 \mathrm{~min}$ & $1029.2827 ; \Delta=-4.220$ & $\mathrm{C}_{48} \mathrm{H}_{53} \mathrm{O}_{25}^{+}$ & Malvidin-3-O-glu-ethyl- malvidin-3-O-glu & 867,705 \\
\hline \multicolumn{5}{|l|}{$\mathrm{E}$} \\
\hline $59-61 \mathrm{~min}$ & $1097.2872 ; \Delta=-4.492$ & $\mathrm{C}_{55} \mathrm{H}_{53} \mathrm{O}_{24}^{+}$ & Malvidin-3-O-glu-ethyl-dimeric procyanidin & 935 \\
\hline $62-63 \mathrm{~min}$ & $1125.3197 ; \Delta=-4.313$ & $\mathrm{C}_{57} \mathrm{H}_{57} \mathrm{O}_{24}{ }^{+}$ & $\begin{array}{l}\text { Malvidin-3-O-glu-ethyl-(epi)catechin-ethyl- } \\
\text { (epi)catechin }\end{array}$ & 963,673 \\
\hline
\end{tabular}

Table 3 Content of native anthocyanins in red wines expressed as $\mathrm{mg} / \mathrm{L}$

\begin{tabular}{lcl}
\hline & Red wine (ctrl) & $\begin{array}{l}\text { Red } \\
\text { wine }+ \text { acet- } \\
\text { aldehyde }\end{array}$ \\
\hline Dp3glc & $39.05 \pm 1.46$ & $\operatorname{tr}$ \\
Cy3glc & $2.82 \pm 0.13$ & $\operatorname{tr}$ \\
Pt3glc & $49.68 \pm 1.07$ & $\operatorname{tr}$ \\
Pn3glc & $22.59 \pm 1.01$ & $\operatorname{tr}$ \\
Mv3glc & $266.77 \pm 14.02$ & $16.55 \pm 0.25$ \\
Pn3acglc & $8.60 \pm 0.40$ & $\operatorname{tr}$ \\
Mv3acglc & $5.84 \pm 0.20$ & $\operatorname{tr}$ \\
Mv3cmglc & $34.54 \pm 0.43$ & $\operatorname{tr}$ \\
\hline
\end{tabular}

Dp3glc $=$ delphinidin 3- $O$-glucoside, Cy3glc $=$ cyanidin 3- $O$-glucoside, $\mathrm{Pt} 3 \mathrm{glc}=$ petunidin 3-O-glucoside, $\mathrm{Pn} 3 \mathrm{glc}=$ peonidin $3-O$-glucoside, $\mathrm{Mv} 3 \mathrm{glc}=$ malvidin 3-O-glucoside, Pn3acglc $=$ peonidin $3-\left(6^{\mathrm{II}}-\right.$ acetyl $)-O$-glucoside, Mv3acglc $=$ malvidin 3-(6II-acetyl $)-O$-glucoside, $\mathrm{Mv} 3 \mathrm{cmglc}=$ malvidin $3-\left(6^{\mathrm{II}}-\right.$ coumaroyl $)-O$-glucoside, $\operatorname{tr}=$ trace

From a quantitative standpoint, analyses on the identified compounds were not reliable as we were not able to precisely assess the potential loss of analytes following the semi-preparative HPLC separation of the analyzed wines and the successive concentration of the obtained fractions. However, some deductions of some interest for enologists in regards to wine production and aging were inferred.

On the basis of our experimental evidence, it was proposed that the anthocyanin moiety is not preferentially attacked by the protonated acetaldehyde, which seems to react first and preferentially with flavanols due to their better nucleophilicity when compared to anthocyanins.
Subsequently, after the loss of a water molecule, the acetaldehyde-flavanol adduct attacks either another flavanol or an anthocyanin unit. In our studies conducted on real wines $(\mathrm{pH}=3.69)$, we only detected polymers containing just one anthocyanin unit, even if molecules of unreacted malvidin-3-O-glucoside were still present in the analyzed samples. On the contrary, flavanol-ethyl-flavanol adducts were detected. Thus, we could suggest that wine $\mathrm{pH}$ must play a central role in determining the outcome of the polymerization reaction involving acetaldehyde, anthocyanins and flavanols. It is reasonable to hypothesize that a higher $\mathrm{pH}$ renders acetaldehyde a worse electrophile thus causing it to react to a lesser extent with any available nucleophile, including flavanols and anthocyanins. As a consequence, in real wines characterized by milder acidic environments compared to the more acidic model solutions with a $\mathrm{pH}$ value usually around 2 , just one anthocyanin moiety seems to be involved in the formation of ethylidene-bridged flavanol polymers. This is a crucial piece of information, as it implies the importance of properly modulating the ratio between native pigments and flavanols as to regulate the desired quantity of new pigments responsible for the wine coloration.

\section{Chromatic characteristics of wines}

The main spectrophotometric data (abs $420 \mathrm{~nm}$, abs $520 \mathrm{~nm}$ and Abs $620 \mathrm{~nm}$ ) and CIELAB color space using the L* $a * b *$ coordinates were determined to understand if changes due to acetaldehyde addition were perceived by human eyes. The color of wine samples was determined as abs units at wavelength usually used to characterize white (abs $420 \mathrm{~nm}$ ) and 
Table 4 Abs $420 \mathrm{~nm}$ and CIELAB coordinates of white wines

\begin{tabular}{lrr}
\hline & White wine (ctrl) & \multicolumn{1}{l}{$\begin{array}{l}\text { White } \\
\text { wine + acetal- } \\
\text { dehyde }\end{array}$} \\
\hline $\mathrm{Abs}_{420 \mathrm{~nm}}$ & $0.15 \pm 0.00$ & $0.19 \pm 0.00$ \\
$\mathrm{~L}^{*}$ & $77.75 \pm 0.66$ & $77.58 \pm 0.17$ \\
$\mathrm{a}^{*}$ & $1.40 \pm 0.00$ & $1.68 \pm 0.05$ \\
$\mathrm{~b}^{*}$ & $7.10 \pm 0.12$ & $9.78 \pm 0.10$ \\
$\mathrm{C}^{*}$ & $7.20 \pm 0.12$ & $9.95 \pm 0.13$ \\
$\mathrm{H}$ & $78.93 \pm 0.25$ & $80.25 \pm 0.19$ \\
$\Delta \mathrm{E}$ & & $2.78 \pm 0.08$ \\
$\Delta$ hue & & $1.33 \pm 0.39$ \\
\hline
\end{tabular}

Table 5 Color intensity, hue and CIELAB coordinates of red wines

\begin{tabular}{lcc}
\hline & Red wine (ctrl) & Red wine + acetaldehyde \\
\hline Abs $_{420 \mathrm{~nm}}$ & $2.91 \pm 0.00$ & $3.11 \pm 0.10$ \\
Abs $_{520 \mathrm{~nm}}$ & $3.23 \pm 0.01$ & $4.92 \pm 0.15$ \\
Abs $_{620 \mathrm{~nm}}$ & $0.64 \pm 0.01$ & $1.49 \pm 0.05$ \\
Color intensity & $6.78 \pm 0.01$ & $9.52 \pm 0.29$ \\
Hue & $0.90 \pm 0.00$ & $0.63 \pm 0.00$ \\
L* $^{*}$ & $67.08 \pm 0.29$ & $11.15 \pm 0.06$ \\
$\mathrm{a}^{*}$ & $17.83 \pm 0.10$ & $31.38 \pm 0.05$ \\
$\mathrm{~b}^{*}$ & $13.05 \pm 0.13$ & $17.48 \pm 0.05$ \\
$\mathrm{C}^{*}$ & $22.08 \pm 0.13$ & $35.95 \pm 0.06$ \\
$\mathrm{H}$ & $36.20 \pm 0.27$ & $29.10 \pm 0.08$ \\
$\Delta \mathrm{E}$ & & $57.71 \pm 0.35$ \\
$\Delta$ hue & & $-7.10 \pm 0.22$ \\
\hline
\end{tabular}

red (abs 420, abs 520, abs $620 \mathrm{~nm}$ ) wines and by determining the CIELAB coordinates in the $a^{*} b^{*}$ color plane, in which color is indicated along the green-red axis $\left(-a^{*}+a^{*}\right)$ and blue-yellow axis $\left(-b^{*}+b^{*}\right)$.

For white wines, the addition of acetaldehyde determined an increase of abs $420 \mathrm{~nm}$ and $a^{*}$ and $\mathrm{b}^{*}$ coordinates (Table 4). Normally, white wines are inside the area defined by the green (negative $a^{*}$ values) and yellow (positive $b^{*}$ values) color. Therefore, the yellow color component was positively influenced by acetaldehyde to a greater degree. The Chroma values, which define chromatic intensity against pure white, were positively correlated with acetaldehyde treatment. Thus, the wines treated with acetaldehyde exhibited higher $\mathrm{C}^{*}$ and hue values than the untreated wines. The effect observed on color was also significant in terms of abs $420 \mathrm{~nm}$ but the shift was lesser than $0.8 \mathrm{abs}$ units, a value generally considered detrimental for the quality of white wines.

The acetaldehyde addition had effects on the chromatic parameters of red wines as well (Table 5). The color intensity (abs $420 \mathrm{~nm}+$ abs $520 \mathrm{~nm}+$ abs $620 \mathrm{~nm}$ ) increased and hue (abs $420 \mathrm{~nm} / \mathrm{abs} 520 \mathrm{~nm}$ ) decreased as already observed $^{9}$. A dramatic increase occurred in the chroma values $\left(C^{*}{ }_{a b}\right)$, while the decrease in the lightness $\left(L^{*}\right)$ was up to 50 CIELAB units. The greater decrease in lightness observed in the case of malvidin-3-O-glucoside should, therefore, be interpreted in terms of a greater coloration of wine treated with acetaldehyde. In the wine treated with acetaldehyde, the hue dropped to lower values and wines showed higher bluer hues as expected [11].

\section{Conclusions}

Although many studies have been performed on the reactivity of acetaldehyde in wine model solutions, quite poor are the reports on the fate of such molecule in real wines. Wines are complex chemical matrices including several compounds often involved in mutual reactions and equilibria. Thus, investigating the fate of natural metabolites in wines can prove challenging. The study we conducted, by providing insights into the chemical response to acetaldehyde of both white and red wines, constitutes a significant step forward towards the understanding of the chemical bases of the wine stability during the aging. More specifically, in white wines we ascertained the massive formation of vinyl-flavan-3-ol derivatives, while in red wines the formation of ethylidenebridged red pigments was predominant. These latter positively enhanced the color properties of red wines and consequently their stability over time; while in white wines flavan-3-ols prevented acetaldehyde from inducing undesired browning effects as well as to reactions that ultimately affect the quality of wine by the production of off-flavors.

The outcome of our research can be of some interest to enologists, since on such knowledge winemakers can hinge the implementation of appropriate technological practices, to opportunely modulate the natural chemical composition of wine metabolites as to guarantee stable high-quality products over time, without having to resort to undesirable external additives.

Finally, the improvement of the color observed in red wines after the addition of an excess of acetaldehyde might be of some interest even to the dyeing industry for the production of stable and intense colorants by reusing wine byproducts quite rich in anthocyanins and tannic pigments.

Acknowledgements The technical assistance of Anna Russo and of all the staff is gratefully acknowledged.

Funding Open access funding provided by Università degli Studi di Napoli Federico II within the CRUI-CARE Agreement. 


\section{Declarations}

Conflict of interest The authors declare that they have no conflict of interest.

Compliance with ethics requirements This article does not contain any studies with human or animal subjects.

Open Access This article is licensed under a Creative Commons Attribution 4.0 International License, which permits use, sharing, adaptation, distribution and reproduction in any medium or format, as long as you give appropriate credit to the original author(s) and the source, provide a link to the Creative Commons licence, and indicate if changes were made. The images or other third party material in this article are included in the article's Creative Commons licence, unless indicated otherwise in a credit line to the material. If material is not included in the article's Creative Commons licence and your intended use is not permitted by statutory regulation or exceeds the permitted use, you will need to obtain permission directly from the copyright holder. To view a copy of this licence, visit http://creativecommons.org/licenses/by/4.0/.

\section{References}

1. Prete P, Fiorentino A, Rizzo L, Proto A, Cucciniello R (2021) Review of aminopolycarboxylic acids-based metal complexes application to water and wastewater treatment by (photo-)Fenton process at neutral pH. Curr Opi Green Sustain Chem 100451

2. Silva Ferreira AC, Barbe JC, Bertrand A (2002) Heterocyclic acetals from glycerol and acetaldehyde in port wines: evolution with aging. J Agric Food Chem 50:2560-2564

3. Sheridan MK, Elias RJ (2015) Exogenous acetaldehyde as a tool for modulating wine color and astringency during fermentation. Food Chem 177:17-22

4. Drinkine J, Lopes P, Kennedy JA, Teissedre PL, Saucier C (2007) Ethylidene-bridged flavan-3-ols in red wine and correlation with wine age. J Agric Food Chem 55:6292-6299

5. Dallas C, Ricardo-da-Silva JM, Laureano O (1996) Products formed in model wine solutions involving anthocyanins, procyanidin B2, and acetaldehyde. J Agric Food Chem 44:2402-2407

6. Doco T, Es-Safi NE, Cheynier V, Moutounet M (1996) Study of the acetaldehyde induced polymerisation of flavan-3-ols by liquid chromatography-ion spray mass spectrometry. J Chromatogr A 752:85-91

7. Es-Safi NE, Fulcrand H, Cheynier V, Moutounet M (1999) Competition between $(+)$-catechin and (-)-epicatechin in acetaldehyde-induced polymerization of flavanols. J Agric Food Chem 47:2088-2095

8. Es-Safi NE, Fulcrand H, Cheynier V, Moutounet M (1999) Studies on the acetaldehyde-induced condensation of (-)-epicatechin and malvidin 3-O-glucoside in a model solution system. J Agric Food Chem 47:2096-2102

9. Sheridan MK, Elias RJ (2016) Reaction of acetaldehyde with wine flavonoids in the presence of sulfur dioxide. J Agric Food Chem 64:8615-8624

10. Morata A, Calderón F, González MC, Gómez-Cordovés MC, Suárez JA (2007) Formation of the highly stable pyranoanthocyanins (vitisins A and B) in red wines by the addition of pyruvic acid and acetaldehyde. Food Chem 100:1144-1152

11. Forino M, Picariello L, Lopatriello A, Moio L, Gambuti A (2020) New insights into the chemical bases of wine color evolution and stability: the key role of acetaldehyde. Eur Food Res Tech 246:733-743

12. Cruz L, Bras NF, Teixeira N, Fernandes A, Mateus N, Ramos MJ, Rodríguez-Borges J, de Freitas V (2009) Synthesis and structural characterization of two diasteroisomers of vinylcatechin dimers. J Agric Food Chem 57:10341-10348

13. Fulcrand H, Benabdeljalil C, Rigaud J, Cheynier V, Moutounet M (1998) A new class of wine pigments generated by reaction between pyruvic acid and grape anthocyanins. Phytochemistry 47:1401-1407

14. Oliveira J, De Freitas V, Mateus N (2009) A novel synthetic pathway to vitisin B compounds. Tetrahedron Lett 50:3933-3935

15. Rentzsch M, Schwarz M, Winterhalter P, Blanco-Vega D, Hermosín-Gutiérrez I (2010) Survey on the content of vitisin A and hydroxyphenyl-pyranoanthocyanins in Tempranillo wines. Food Chem 119:1426-1434

16. Jenkins TW, Howe PA, Sacks GL, Waterhouse AL (2020) Determination of molecular and "Truly" free sulfur dioxide in wine: a comparison of headspace and conventional methods. Am J Enol Vitic 71:222-230

17. Organisation Internationale de la Vigne et du Vin (2020) Compendium of international methods of wine and must analysis https:// www.oiv.int (archived on 13 Dec 2020)

18. Bravo MN, Silva S, Coelho AV, Boas LV, Bronze MR (2006) Analysis of phenolic compounds in Muscatel wines produced in Portugal. Anal Chim Acta 563:84-92

19. Frost S, Lerno LA, Zweigenbaum J, Heymann H, Ebeler SE (2018) Characterization of red wine proanthocyanidins using a putative proanthocyanidin database, amide hydrophilic interaction liquid chromatography (HILIC), and time-of-flight mass spectrometry. Molecules 23:2687

20. Pons A, Lavigne V, Landais Y, Darriet P, Dubourdieu D (2010) Identification of a sotolon pathway in dry white wines. J Agric Food Chem 58:7273-7279

21. Ma L, Waterhouse AL (2018) Flavanols react preferentially with quinones through an electron transfer reaction, stimulating rather than preventing wine browning. Anal Chim Acta 1039:162-171

22. Laitila JE, Suvanto J, Salminen JP (2019) Liquid chromatography-tandem mass spectrometry reveals detailed chromatographic fingerprints of anthocyanins and anthocyanin adducts in red wine. Food Chem 294:138-151

23. Bakker J, Timberlake CF (1997) Isolation, identification, and characterization of new color-stable anthocyanins occurring in some red wines. J Agric Food Chem 45:35-43

24. Atanasova V, Fulcrand H, Le Guernevé C, Cheynier V, Moutounet M (2002) Structure of a new dimeric acetaldehyde malvidin 3-glucoside condensation product. Tetrahedron Lett 43:6151-6153

Publisher's Note Springer Nature remains neutral with regard to jurisdictional claims in published maps and institutional affiliations. 